\title{
Rencontre avec Hugues Hotier, directeur de la revue Communication \& Organisation
}

\section{OpenEdition}

\section{Journals}

Édition électronique

URL : http://journals.openedition.org/communicationorganisation/2608

DOI : 10.4000/communicationorganisation.2608

ISSN : 1775-3546

Éditeur

Presses universitaires de Bordeaux

\section{Édition imprimée}

Date de publication : 1 novembre 2001

ISSN : 1168-5549

Référence électronique

"Rencontre avec Hugues Hotier, directeur de la revue Communication \& Organisation », Communication et organisation [En ligne], 20 | 2001, mis en ligne le 27 mars 2012, consulté le 19 avril 2019. URL :

http://journals.openedition.org/communicationorganisation/2608; DOI : 10.4000/ communicationorganisation.2608

Ce document a été généré automatiquement le 19 avril 2019

(c) Presses universitaires de Bordeaux 


\section{Rencontre avec Hugues Hotier, directeur de la revue Communication \& Organisation}

1 Ce numéro 20 de la revue Communication et Organisation nous a semblé suffisamment particulier, pour ne pas dire exceptionnel, pour que nous vienne l'idée de consacrer la rubrique "Entrevue» à son fondateur et directeur scientifique, Hugues Hotier. Les questions qui lui sont posées sont anonymes et nous avons réuni leurs auteurs sous la formulation neutre C. \& O., abréviation de Communication \& Organisation.

2 C. \& O.: Hugues Hotier, vous avez fondé le Groupe de Recherche en Communication des Organisations il y a une quinzaine d'années et la revue Communication \& Organisation il y ajuste dix ans. Pouvez-vous nous en dire plus sur les motivations qui poussent un universitaire à de telles démarches.

Hugues Hotier: Quand je suis arrivé à Bordeaux 3, en janvier 1985, je venais d'une université de technologie où la recherche collective était très développée. Il y avait des laboratoires avec des équipes centrées sur des projets communs. Souvent, les recherches qu'on y menait étaient commanditées et nécessitaient un fonctionnement du type conduite de projet. Les thèses s'inscrivaient dans les programmes de recherche et $\mathrm{y}$ contribuaient. Il y avait une sorte de vie commune autour d'un objectif et cela s'inscrivait dans la durée. Ma nomination à Bordeaux 3 me faisait renouer avec un monde que j'avais perdu de vue depuis que j'avais terminé mes études, celui des universités littéraires. Je passerai sur mon désappointement devant le conservatisme ambiant. J'avais fait toute ma carrière dans des structures dont la technologie était l'objet : un lycée technique, un IUT, une école d'ingénieurs. J'avais parfois l'impression, à Bordeaux 3, que mes collègues n'avaient pas conscience des changements que connaissait l'environnement et qu'ils considéraient l'université comme une espèce d'abri atomique qui les protégeait des contaminations extérieures. Je m'empresse de dire, pour atténuer mon propos, qu'il n'est pas impossible que les choses aient changé. Ce qu'on appelle centre de recherche dans les disciplines qui ressortissent aux lettres et aux sciences humaines n'est souvent qu'une juxtaposition de chercheurs qui produisent en solitaire. On pourrait d'ailleurs dire à peu près la même chose des sciences juridiques, économiques et politiques. Leur production 
est souvent d'un excellent niveau mais la notion d'équipe relève la plupart du temps de la fiction. Dans les universités de sciences ou de technologie, il faut de tels investissements pour installer un laboratoire que l'amortissement ne peut se concevoir que dans une démarche collective. Quand on connaît les conditions déplorables que l'Université fait aux chercheurs en lettres et sciences humaines on comprend qu'ils préfèrent travailler chez eux. L'objet des réunions relève plus souvent de l'information que de la collaboration. On se tient au courant des travaux que l'on mène plutôt qu'on ne mène des travaux ensemble. Je reconnais volontiers que les choses ont évolué et que le tableau n'est plus aussi noir qu'il l'était il y a dix-sept ans. À Compiègne, j'avais travaillé en solitaire. D'abord parce qu'il n'y avait pas, à l'époque, de recherche en sciences humaines et sociales; ensuite parce que, préparant mon doctorat d'état, j'étais centré sur mon projet personnel et peu soucieux de me distraire de cette recherche lourde et longue. Quand j'ai pris la succession de Robert Escarpit à Bordeaux 3, j'ai apporté ma compétence en communication organisationnelle et développé la recherche, la formation aussi, dans ce domaine. Il n'y avait pas de recherche en communication des organisations, j'ai pensé qu'il était de mon devoir d'y remédier.

4 Quant à la revue, sa création relève d'une démarche complémentaire. Il me paraît indispensable de développer ce qu'on appelle la valorisation de la recherche. Pour cela, il faut que soient multipliées les occasions de rencontre et de confrontation. C'est l'un des objectifs qu'on peut assigner aux colloques, séminaires et autres journées d'étude. Il faut aussi qu'existent des possibilités de publication pour que les chercheurs puissent diffuser leurs travaux le plus largement. Et pas seulement les chercheurs connus et reconnus, les jeunes aussi. Avant Communication \& Organisation, il n'existait pas de revue de langue française dédiée à la communication des organisations. Il fallait en créer une. Nous l'avons fait et le premier numéro est sorti en mai 1992. Je me souviens des prophéties pessimistes qui accueillirent, en décembre 1991, l'annonce du projet de création de la revue. On me prédisait que ce serait un travail considérable pour une entreprise non viable et qui tournerait court. Sur le premier point, la prédiction fut amplement vérifiée. Pour le second point, je suis plus réservé car on ne peut pas dire que dix ans d'existence ne constituent pas une durée de vie convenable pour une revue de recherche. Et ce n'est pas fini, nous avons en chantier des numéros jusqu'en décembre 2003...

C. \& 0. : On comprend que la réalisation d'une revue telle que la nôtre ne soit pas un travail facile. La gestion en est-elle plus aisée?

6 Hugues Hotier : Certainement pas! Un jour que je disais à Denis Pryen, le directeur des éditions L'Harmattan que notre revue ne tirait qu'à 250 exemplaires, il m'en félicita, ajoutant que sa longévité exceptionnelle était un indicateur de réussite. Si vous éditez une revue qui s'appelle Sciences humaines, vous disposez d'un lectorat potentiel non négligeable. Ce n'est pas Paris Match mais vous pouvez quand même vendre en kiosque. Si votre revue s'appelle Communication, cela diminue déjà le public concerné. Mais si vous précisez Communication \& Organisation, vous réduisez encore ce que les gens du marketing appellent le public-cible... Imaginons qu'entre les universitaires spécialisés dans la communication organisationnelle et leurs étudiants il y ait en France 10000 lecteurs potentiels. Ce n'est pas si mal, d'autant que le monde de l'entreprise a, à de rares exceptions près, d'autres lectures. Considérez que les bibliothèques universitaires - elles sont presque toutes abonnées - satisfont la majeure partie de ce lectorat. En d'autres termes, 250 exemplaires pour une revue semestrielle comme la nôtre, inscrite au 
catalogue de beaucoup de bibliothèques, c'est bien. Même si c'est peu pour équilibrer le budget.

7 C. \& 0.: Il est équilibré? Plus généralement, quels sont les revenus d'un groupe de recherche comme celui que vous dirigez?

8 Hugues Hotier: Evidemment, la revue est déficitaire. Je ne sais d'ailleurs pas s'il y a beaucoup de revues de recherche qui puissent équilibrer leurs comptes. Mais ce n'est pas important. Il faut gérer au plus près et trouver les moyens de diffuser une revue qui permet à des jeunes chercheurs de voisiner avec des personnalités reconnues. Disons, pour simplifier ces comptes d'apothicaire, que les abonnements permettent de payer un numéro, diffusion comprise. L'autre numéro voit le jour grâce à un mécène (devrais-je dire un sponsor?) qui nous aide et qui ne souhaite pas trop que nous le remerciions publiquement de peur de voir venir à lui un cortège d'universitaires affamés. La dotation que nous recevons du ministère est de 16000 francs pour le fonctionnement (les frais de secrétariat sont largement supérieurs à cette somme!) et de 6000 francs pour l'équipement. Sans commentaires. Ou plutôt si, un commentaire. Le GREC/O est l'un des trois laboratoires qui forment le Centre d'Étude des Médias. Lequel perçoit une somme qu'il partage en trois. Si le GREC/O était un centre à lui seul, il percevrait la totalité. Et il en serait de même pour chacun des deux autres labos. Mais le ministère exige des regroupements, allez savoir pourquoi... Probablement pour des raisons épistémologiques... Cela dit, le GREC/O réussit depuis une dizaine d'années à payer les salaires de deux collaboratrices. L'une assure le secrétariat et gère les abonnements de la revue. L'autre se charge de la réalisation matérielle de la revue. À elles deux, elles travaillent l'équivalent d'un temps et demi. On se débrouille. On passe une bonne partie du temps à chercher des contrats et à faire rentrer de l'argent. C'est une autre forme de recherche... Disons que le management de la recherche est une condition de l'existence même d'un laboratoire. Ainsi, par exemple, la décision de ne pas payer de missions aux membres du groupe et, plus encore, aux doctorants, n'a pas été facile à prendre. Et pas facile à annoncer. Mais, c'est cela le mandarinat, non? Et puis, en même temps que le vingtième numéro de notre revue, nous fêtons le quinzième anniversaire d'un groupe de recherche dont l'acte de naissance a été le premier colloque Entreprise et communication que nous avons organisé en novembre 1986 ! Alors, ne nous plaignons pas.

C. \& O. : Laissons de côté ces questions d'argent. Nous savons bien que les chercheurs sont de purs esprits. Pouvez-vous nous parler de la recherche telle qu 'elle existe au GREC/O?

Hugues Hotier: Sur le plan de l'organisation, il faut savoir que si le GREC/O est une composante de l'Université Michel de Montaigne-Bordeaux 3, les chercheurs qui le composent viennent de plusieurs universités et de plusieurs régions : Bordeaux 1, Calais, Paris, Reims, Tours.

Il n'est pas facile de les rassembler. Nous organisons deux séminaires longs chaque année, l'un au printemps, l'autre à l'automne. Le reste du temps, nous fonctionnons selon les principes du télétravail. Pour la nature même de nos recherches, nous fonctionnons selon un rythme triennal. Nous choisissons ensemble un thème de recherche pour trois ans. À l'issue de la deuxième année, nous publions un ouvrage collectif qui est aussi conçu comme les textes préparatoires d'un colloque qui clôturera la recherche un an plus tard. Une année après le colloque, nous en publions les actes. À ce moment, nous sommes déjà dans la thématique suivante. L'idée est de trouver un thème qui permette une réflexion et une production communes et, en même temps, une déclinaison personnelle. Chacun des membres du groupe doit pouvoir décliner le thème en fonction de ses intérêts personnels 
et publier ses propres travaux démarqués des recherches collectives. Par exemple, quand nous avons traité le non-verbal dans les organisations, nous avons publié un ouvrage commun mais les déclinaisons personnelles étaient aussi diverses que riches. L'un travaillait sur les emblèmes et l'identité visuelle des entreprises, un autre sur les rythmes qui structurent les discours, un autre encore sur les postures, etc. La thématique actuelle a pour titre « coexister dans les organisations ». Notre prochain colloque aura lieu en mai 2003.

C. \& 0.: Quand on inventorie les thématiques qui ont été les vôtres depuis quinze ans, peut-on parler d'un paradigme qui serait celui du GREC/O. Avez-vous constaté une évolution de votre démarche?

Hugues Hotier: Depuis une dizaine d'années, nous tournons autour d'un concept que nous explorons avec intérêt, j'avais envie de dire avec avidité, que nous appelons l'induction. Nous essayons d'échapper au schéma émetteur-message-récepteur dont, quoi qu'on en dise, on a beaucoup de mal à se départir, pour essayer de comprendre la réalité de la communication, de l'état de communication au sens où l'entend, par exemple, Birdwhistel. Oui, certainement, il y a eu une évolution au sein de notre groupe. Je crois la résumer fidèlement en disant que nous nous sommes peu à peu éloignés du terrain pour nous hisser au niveau des concepts. Nous continuons à observer le terrain mais nous le faisons différemment, nous sommes moins collés au quantitatif. Nous théorisons davantage. C'est le bonheur de l'heuristique.

C. \& O. : Dans vos articles comme dans vos propos vous parlez souvent de bonheur, de plaisir aussi. Quand on vous demande ce que vous faites au GREC/O, vous dites qu'on s'y amuse bien... On pourrait trouver cela curieux.

Hugues Hotier: Vous n'allez pas me dire, après tant d'autres, que la recherche est une souffrance ? La recherche est un plaisir et nous sommes des privilégiés, nous qui sommes payés pour étudier tout au long de notre vie. Dans un article que je n'aurais pas dû lire avant de répondre à cette entrevue, des jeunes chercheurs, des doctorants du GREC/O parlent du principe de plaisir. Vous n'imaginez pas à quel point ces propos me touchent et m'émeuvent. C'est bien que des jeunes entrent dans la carrière en chantant. D'ailleurs, à ce propos, je révèle que nous chantons beaucoup dans nos séminaires. Au sens propre du terme. Nos soirées sont gastronomiques et chantantes. Oui, la recherche est un plaisir. Un plaisir aux origines multiples. Plaisir d'être ensemble, de travailler, de chercher et de produire ensemble. Plaisir de se sentir intelligent. Étonnant? Pas tant que cela. Quand nous nous séparons après une semaine de travail intellectuel, chacun d'entre nous a l'impression de se sentir plus riche, plus apte à observer, découvrir, analyser, interpréter. Plaisir aussi de voir les jeunes heureux parmi nous et avec nous, confiants, enthousiastes et débordants de propositions. Avec nous, ils se familiarisent avec les méthodes dans un sain compagnonnage où le professeur se sent plus ouvrier qualifié formant un apprenti que maître enseignant ses disciples.

\section{C. \& 0. : Vous avez des disciples?}

Hugues Hotier: Certainement pas! Jamais! Cela dit, il y a effectivement un risque d'inféodation du doctorant à son directeur. Parce que la préparation d'une thèse tend à instaurer une relation de confiance quasi filiale. Le risque, ce serait que cette relation amicale et confiante engendre une soumission intellectuelle néfaste. Être disciple, c'est avoir un maître. Il ne faut pas avoir de maître, il faut vivre libre. Je me souviens, quand j'étais étudiant, des professeurs qui évoquaient leurs propres professeurs par des 
formules du type «Mon maitre Lebègue, mon maître Brunschwig ». Aujourd'hui encore, on voit des intellectuels qui se prosternent devant des maittres à penser et qui se comportent devant Edgar Morin ou Pierre Bourdieu comme les lecteurs de FranceDimanche devant Stéphanie de Monaco ou Roch Voisine. On respecte, on admire, mais on ne s'inféode pas. C'est puéril et grotesque. J'ai préparé mes deux thèses sous la direction de Jean-Claude Chevalier qui fut aussi le patron d'Eliseo Veron. Quand il nous arrivait de parler de lui, avec Eliseo, c'était avec respect et tendresse mais nous ne privions pas de le mettre en question. Parce que nous l'aimions trop pour le statufier. Précisément, l'état de disciple, sorte d'esclavage intellectuel librement consenti qui, dans une certaine mesure, s'apparente au masochisme, érode l'esprit critique, anéantit le doute scientifique et empêche la créativité. Si j'avais un message à délivrer aux jeunes intellectuels, aux jeunes chercheurs, notamment aux doctorants, je leur dirais « Doutez de tout, doutez de tous, notamment de ceux que vous respectez. Vous ne les en aimerez que davantage car vous leur aurez rendu leur dimension humaine. » 